\title{
The Effects of Objective and Subjective Knowledge on the Exploratory Acquisition of Wine
}

\author{
Leyland Pitt, Frauke Mattison Thompson, Anne Engstrom, Joe Velle, \\ Adam Mills, and Jan Kietzmann
}

\begin{abstract}
This chapter investigates the effect of objective and subjective knowledge on exploratory acquisition in consumers' purchase of wine. The main research questions investigated are whether there is a link between a consumer's objective knowledge of wine (what they really know) is linked to their subjective knowledge (what they think they know), and whether these impact on the extent to which the consumer engages in exploratory purchasing behavior (tries new brands, tries different wines, etc). The literature for the three constructs is reviewed, and hypotheses are developed. The study then investigated these questions among an online sample of consumers in the USA. Data is analyzed, results are provided, and the implications for management are discussed. Limitations are also noted and directions for future research are indicated.
\end{abstract}

L. Pitt • A. Mills • J. Kietzmann

Simon Frasier University, Burnaby, BC, Canada

e-mail: 1pitt@sfu.ca; adamm@sfu.ca; jkietzma@sfu.ca

F.M. Thompson $(\square)$

King's College London, London, UK

e-mail: frauke.mattison_thompson@kcl.ac.uk
A. Engstrom
Lulea University of Technology, Lulea, Sweden
e-mail: Anne.Engstrom@ltu.se
J. Velle
University of Malta, Msida, Malta
e-mail: joseph.m.vella@um.edu.mt 